\title{
Examining the Dimensions of Intimacy in Twin and Peer Relationships
}

\author{
Andrea K. Foy', Phillip A.Vernon' and Kerry Jang ${ }^{2}$ \\ 'Department of Psychology, The University of Western Ontario, London, Ontario, N6A 5B8, Canada \\ ${ }^{2}$ Department of Psychiatry, 2255 Westbrook Mall, University of British Columbia,Vancouver, British Columbia,V6T 2A I, Canada
}

\begin{abstract}
W hile it is widely assumed that twinship involves high levels of intimacy to the detriment of other relationships, an examination of twinship using measures of intimacy has not yet been conducted. Participants were 214 individual twins (128 MZ, 62 DZ same-sex, and 24 DZ mixed-sex) between 16-73 years of age. Twins completed measures of intimacy in reference to their co-twin, their closest same-sex friend, and their closest other-sex friend. As expected, the highest level of intimacy was reported for the co-twin. Contrary to expectation, $\mathrm{MZ}$ twins did not report significantly higher levels of intimacy with their co-twin, or significantly lower levels of intimacy with their closest friends when compared to DZ same-sex or DZ mixed-sex twins. Furthermore, twins who reported high levels of intimacy with their co-twin did not report significantly lower levels of intimacy with their closest friends. Implications and directions for future research are discussed.
\end{abstract}

Researchers agree that intimacy is a component of all significant relationships (Sharabany, 1994a) and that intimacy, like personality, evolves over time (Jones \& Dembo, 1989). Intimacy needs differ across developmental stages, and these needs are met through a variety of different relationships (Buhrmester \& Furman, 1986). Since development occurs continuously through the relationships held, each relationship shapes current and future interactions with others. Indeed, friendships are shaped by culture, developmental period, personal characteristics, and friendship needs at the time (Sharabany, 1994a/b). With twins, however, it is often assumed that their intimate relationship excludes or discourages outside relationships (Zazzo, 1976). As a result, many researchers believe that twins do not experience the wide variety of relationships necessary for healthy social and psychological development (Clark \& Dickman, 1984). Although a number of intimacy measures exist, researchers have not yet examined how twinship influences the relationships that twins have with others. Rather, assumptions have been based on the amount of time twins spend together, their similar appearance and treatment, and their high degree of social closeness.

If time spent together is an indication of relationship exclusivity, then monozygotic (MZ) twins may be at a particular risk when compared to dizygotic (DZ) twins. Researchers have demonstrated that MZ twins are more likely to share the same room (Pearlman, 1990), spend more years together in school (Tambs et al., 1985), attend the same school longer, be in the same class, separate from their co-twin for the first time significantly later than DZ twins (Pearlman, 1990), indicate being 'inseparable', and be likely to act in unison (Sandbank, 1988). Although Segal (1999) believes that a valid indication of intimacy may be the ability to maintain emotional closeness with another individual despite a lack of contact (i.e., twins reared apart often report high levels of intimacy upon reunion), researchers continue to emphasize the importance and the hazards of shared activities when evaluating the intimate nature of the twin relationship.

Siemon (1980) hypothesized that identical twins may be at a social disadvantage due to their similar appearance and treatment. While the twins and the mothers of twins in Vandenberg's (1984) study did not perceive that twins were treated as a unit or acted as a unit, researchers indicate that when compared with $\mathrm{DZ}$ twins, $\mathrm{MZ}$ twins are more likely to be mistaken for each other (Vandenberg, 1984), to be encouraged by their parents to be alike rather than different, to dress alike, and to have significantly more names that rhyme (Pearlman, 1990). This may become problematic if twins idealize their relationship or identify themselves by their twinship. Adelman and Siemon (1986) assert that "If the twin relationship becomes so satisfying and necessary that it excludes others and interferes with individual growth, it can limit rather than enhance the lives of twins"(p. 98). As a result, researchers predict that twinship may isolate twins from outside relationships, thereby impairing their social adaptation (Zazzo, 1976).

Nevertheless, high levels of intimacy with another individual do not necessarily lead to high levels of dependence (Brothers, 1991); individuation and connectedness may complement rather than compete with one another (Mayseless et al., 1998). Moreover, Collins and Repinski (1994) indicate that current relationships are not abandoned in order to satisfy new ones; both relationships are modified so that they can coexist. Unfortunately, twin researchers have not examined whether or not twins maintain a close relationship with their co-twins in addition to close relationships with others. Rather, researchers have assumed that twins hold exclusive relationships with their co-twins at the expense of forming close relationships with others.

Address for correspondence: Andrea K. Foy, Department of Psychology, The University of Western Ontario, London, Ontario, N6A 5C2, Canada.E-mail: akfoy@uwo.ca 
Where some researchers predict problems, however, others have not found empirical support for the claim that twins are at a disadvantage when compared to singletons. Pearlman's (1990) comparative study between twins (MZ and DZ) and singletons over the age of 18 years,

... did not support the popular notion that twins face special problems with respect to personality development or the idea that twins have more difficulty than others in establishing and maintaining close relationships... Twins and nontwins did not differ significantly with respect to marital status, number of years married, whether married before, number of previous marriages, number of years divorced, or living arrangements if single (p. 619).

Consequently, it is unclear whether or not: 1) twins experience more problems than singletons, 2) these problems are related to twinship, or 3) these problems disappear with age.

Finally, some researchers have theorized that the intimate experiences that twinship brings and the skills that twins learn can be generalized to other healthy relationships (Clark \& Dickman, 1984). Perhaps, then, highly intimate relationships are only detrimental when they are accompanied by extremes in specific areas. Therefore, high risk dimensions need to be identified as well as adverse trends for intimacy scores in order to identify twins who are at risk versus twins who benefit from such a close, stable relationship. In fact, it is possible that certain dimensions of intimacy between twins lead to more intimate peer relationships.

Although researchers have made assumptions regarding intimacy in twinship and how this closeness may influence twins' relationships with others, an examination of twinship using measures of intimacy has not yet been done. Furthermore, the research has not examined how the various dimensions of intimacy differ as a function of zygosity, age, sex, or relationship type. The current study will attempt to answer these questions by examining twins' perceived levels of intimacy with their closest same-sex (SS) friend, closest other-sex (OS) friend and their co-twin, using the Sharabany Intimacy Scale (Sharabany, 1974). Through an examination of total intimacy scores, and scores on each of this scale's eight dimensions, it will be possible to determine how each element contributes to these three relationships, and whether or not the intimacy trends observed in singletons will be reported by twins.

\section{Materials and Method Participants}

Participants were 214 individual twins (158 female, 56 male), $16-73$ years of age $(M=33.64, S D=14.53)$. There were a total of $128 \mathrm{MZ}$ (100 female, 28 male), $62 \mathrm{DZ}$ ss ( 46 female, 16 male), and 24 DZ ms (12 female, 12 male) twins. Participants were recruited from London, Ontario (44\%) and Vancouver, British Columbia (56\%) in Canada through newspaper advertisements $(72 \%)$, personal contacts $(20.6 \%)$, other participants $(2.8 \%)$, and twin clubs $(0.9 \% ; 3.7 \%$ did not indicate where they heard about the study). All twins were initially asked whether they and their co-twin would be interested in participating in a study on twins' relationships with each other and with their best friends. The majority of the participants were single, between 20-35 years of age, well educated, and employed full time (see Table 1). The response rate for returned questionnaires was moderate; $68 \%(n=47)$ complete pairs from London and 43\% $(n=60)$ complete pairs from Vancouver (this rate is based on the number of questionnaires sent to twin pairs and the number of complete pairs returned before data entry was complete). In an attempt to avoid the sample bias typical of twin volunteers (more MZ twins and more females), all participants were given $\$ 10$ once their completed questionnaires were returned.

\section{Measures}

Twins completed the Diagnosis of Twin Zygosity (Nichols \& Bilbro, 1966), the Sharabany Intimacy Scale (Sharabany, 1974), and a battery of other measures that are not pertinent to this report. Each twin completed three versions of the Sharabany Intimacy Scale with reference to their relationship with their closest SS friend, their closest OS friend and their co-twin. Participants were asked not to include relatives or romantic partners as their closest SS or OS friends. Of the 214 participants, $97.2 \%$ indicated that they had a closest SS friend (none of whom were romantic partners), and $77.1 \%$ indicated that they had a closest OS friend, of whom $2.3 \%$ were romantic partners at the time. Ratings for romantic OS friends were not included in any of the analyses.

Participants were also asked, "Who do you consider to be your closest friend?", and were given the following response options: twin, same-sex best friend, opposite-sex best friend, or other. Reciprocity between twin pairs was determined by examining who twins nominated as their closest friend. Three categories of reciprocity emerged for

\section{Table 1}

Percent and Frequency of Participants in Demographic Categories

\begin{tabular}{lcc}
\hline & Percent (\%) & Frequency $(n)$ \\
\hline Age Category & & \\
Adolescence (<20 years of age) & 18.7 & 40 \\
Young Adulthood (20-35 years of age) & 40.2 & 86 \\
Middle Adulthood (36-50 years of age) & 25.2 & 54 \\
Older Adulthood (> 50 years of age) & 15.9 & 34 \\
Marital Status & & \\
Single & 51.4 & 110 \\
Married/Common Law & 41.1 & 88 \\
Divorced & 5.6 & 12 \\
Widowed & 1.9 & 4 \\
Education & & \\
Grade 12 or less & 18.7 & 40 \\
High School Diploma & 23.8 & 51 \\
College/Undergraduate Degree & 35.0 & 75 \\
Professional Training & 13.0 & 28 \\
Graduate Degree & 4.2 & 9 \\
Post Graduate Degree & 5.1 & 11 \\
Employment Status & & \\
Employed Full Time & 39.7 & 85 \\
Employed Part Time & 20.6 & 44 \\
Student & 30.8 & 66 \\
Unemployed & 8.4 & 18 \\
Missing & .5 & 1 \\
\hline
\end{tabular}


each twin pair: 1) Both, when twins chose their co-twin as their closest friend and their co-twin chose them, 2) One, when twins chose their co-twin as their closest friend, but their co-twin did not choose them, and 3) Neither, when twins did not choose their co-twin as their closest friend and their co-twin did not choose them. Note that the Both category of reciprocity did not include twins who mentioned their co-twin and another individual as their closest friend (this type of reciprocity was believed to be different from the reciprocity between twins where other individuals were not involved).

\section{Sharabany Intimacy Scale}

The Sharabany Intimacy Scale (Sharabany, 1974) is a multidimensional self-report measure that evaluates eight distinct, yet related components of intimacy based on sociological, social psychological and psychoanalytic literature: Frankness/Spontaneity, Sensitivity/Knowing, Attachment, Exclusiveness, Giving/Sharing, Imposing/Taking, Common Activities, and Trust/Loyalty (Sharabany, 1974; Sharabany et al., 1981). This particular questionnaire was chosen due to its ability to generate a global indicator of intimacy (total intimacy), as well as individual scores for the eight dimensions. The dimension scores are important when determining specific trends among intimacy components as well as how these components relate to, or predict the variety of relationships held. The original version of the Sharabany Intimacy Scale includes 32 items (4 items on each dimension). However, due to an error in printing, participants in the current study completed only 27 of the 32 items. The dimensions with missing items include Giving/Sharing, Imposing/Taking, and Common Activities.

Participants used a 7-point Likert scale ranging from 1 (Never) to 7 (Always) to indicate how much each item reflected their relationship with their closest SS friend, closest OS friend and co-twin. Dimension scores were generated by computing the average score across the items included in each dimension. Total intimacy scores were produced by calculating the average score across each of the eight dimensions (Sharabany, 1974). Higher levels of intimacy were reflected by higher dimension scores and higher total intimacy scores. For some analyses, twins were classified according to their total intimacy scores for their co-twin, where those who scored between 6-7 were categorized as High, those who scored between 4-5 were categorized as Average, and those who scored between 1-3 were categorized as Low. In most cases, missing scores were replaced by each individual's average score across the remaining items on that dimension. Scores were not replaced if more than $50 \%$ of the items were left unanswered for that individual on the relevant dimension.

The Sharabany Intimacy Scale (Sharabany, 1974) has demonstrated strong psychometric properties across a wide variety of populations, relationships, and developmental stages (reviewed in Sharabany, 1994a). More specifically, this scale has high levels of internal consistency in child, adolescent, and adult populations across a variety of relationships (Mayseless, 1993; Mayseless et al., 1997; Mayseless et al., 1998). The current study supports this with high item-total correlations for each of the three relationship types (Cronbach alphas; .88, .91, .93, for closest SS friend, closest OS friend, and co-twin, respectively). Internal consistency across the eight components of intimacy varied from moderate to high, with Cronbach alphas ranging from .50-.85 in each of the three relationship types.

\section{Diagnosis of Twin Zygosity}

Zygosity was determined with Nichols and Bibro's (1966) Diagnosis of Twin Zygosity. This questionnaire includes five items that assess genetically influenced characteristics, such as eye color, hair color and texture, height, and weight. In addition, participants were asked how often parents, close friends, teachers and acquaintances confuse them with their co-twin. Finally, each twin was asked to indicate and explain whether or not they have had any experiences or illness that their twin has not had. These items helped to differentiate twins with different physical characteristics that arose from accidents, illness, or environmental factors. When compared with blood typing analysis, this measure has a reported accuracy rate of 95\% (Kasriel \& Eaves, 1976).

\section{Procedure}

Once interested twins contacted the researchers, they were each mailed a questionnaire package to complete in the privacy of their own home. This package included a cover letter to inform participants that their co-twin had been sent an identical package and that they were to complete their questionnaires independently. Twins were asked not to discuss their responses to the questionnaire items with their co-twin until after they had both returned their packages. Participants were also asked to read and sign an informed consent form before completing any other forms for the study. Parental consent was requested for twins under 18 years of age. Each participant was provided with a preaddressed postage paid envelope to return the study materials. Once the completed packages were returned, each London twin received a feedback form as well as $\$ 10$ in appreciation for their time (twins in Vancouver were entered into a draw for a chance to win a $\$ 500$ cash prize). The opportunity to receive a summary of the study's results was also given.

\section{Results}

\section{Testing Intimacy Hypotheses}

Total Intimacy Scores for Co-twin, Closest SS, and Closest OS Friend

Two paired $t$ tests were performed to test the hypothesis that the highest level of total intimacy would be reported with co-twin, followed by intimacy with closest SS friend and closest OS friend respectively. The first test found that total intimacy scores for co-twin $(M=5.28)$ were significantly higher than total intimacy scores for closest SS friend $[M=4.82 ; t(201)=6.59, p<.001]$. A significant (albeit low) positive correlation was found between total intimacy scores with co-twin and closest SS friend $(r=.17, p<.05)$, indicating that as intimacy with co-twin increased, intimacy with closest SS friend also increased. 
A second paired $t$ test found that total intimacy scores with closest SS friend $(M=4.90)$ were significantly higher than total intimacy scores with closest OS friend $[M=4.51$; $t(155)=6.05, p<.001]$. A significant positive correlation was found between total intimacy scores with co-twin and closest OS friend $(r=.42, p<.001)$, indicating that higher levels of reported intimacy with co-twin were related to higher levels of reported intimacy with closest OS friend.

\section{Total Intimacy Scores as a Function of Zygosity and Sex}

A MANOVA was performed with Zygosity and Sex as the independent variables and total intimacy scores with cotwin, closest SS friend, and closest OS friend as the dependent variables. The purpose of this analysis was to test whether there was a significant interaction between Zygosity and Sex on total intimacy scores. It was predicted that $M Z$ twins and females would report significantly higher levels of total intimacy with their co-twin when compared to DZ (ss and ms) twins and males, but that MZ twins and males would report lower levels of intimacy with closest SS and closest OS friend. Significant multivariate effects were found for Sex [Pillai's Trace $=.06, F(3,147)=$ 3.21, $p<.05, \eta^{2}=.06$ ], and Zygosity [Pillai's Trace = .09, $\left.F(6,296)=2.39, p<.05, \eta^{2}=.05\right]$, but not for the interaction between Zygosity and Sex [Pillai's Trace $=.07, F(6$, 296) $\left.=1.87, p=.09, \eta^{2}=.04\right]$. At the univariate level, a significant main effect of Sex on total intimacy scores with closest SS friend was found $\left[F(1,149)=4.47, p<.05, \eta^{2}\right.$ $=.03]$, indicating that females reported significantly higher levels of intimacy with their closest SS friend $(M=4.95)$, when compared to males $(M=4.78)$. A main effect of Sex was not found for total intimacy scores with co-twin or closest OS friend. Although a significant effect of Zygosity was found at the multivariate level, it was not observed at the univariate level $\left[F(3,147)=3.21, p<.05, \eta^{2}=.06\right]$ indicating that $\mathrm{MZ}, \mathrm{DZ}$ ss and $\mathrm{DZ} \mathrm{ms}$ twins did not report significantly different levels of intimacy in any of the three relationships.

\section{Reciprocity}

When participants were asked to indicate who they considered their closest friend to be, 64\% $(n=137)$ indicated their co-twin, $20.6 \%(n=44)$ indicated their closest SS friend, $5.6 \%(n=12)$ indicated their closest OS friend, $8.9 \%(n=19)$ indicated other, and $.9 \%(n=2)$ did not respond. Twins who chose their co-twin as their closest friend in addition to another individual were included in the "Other" category since this relationship was deemed to be different from relationships where co-twin was the only friend listed. Only 2.3\% $(n=5)$ of the sample listed twin and other as closest friend. Reciprocity between twin pairs was measured by examining to what extent twins nominated their co-twin as their closest friend. Overall, 51.4\% ( $n=$ $110)$ chose their co-twin as their closest friend and their cotwin chose them (Both), 23.4\% $(n=50)$ chose their co-twin, but their co-twin did not choose them (One), and $23.4 \%(n=50)$ did not choose their co-twin as their closest friend and their co-twin did not choose them (Neither).

A chi-square analysis was performed to determine whether there was an association between the frequency with which twins chose their co-twin as closest friend and their zygosity, where $\mathrm{MZ}$ twins were predicted to choose their co-twin as closest friend significantly more often than $\mathrm{DZ}$ ss or DZ ms twins. The results indicate that there was a significant association between level of reciprocity with cotwin and zygosity $\left[\chi^{2}(4)=55.68, p<.001\right]$, where members of a MZ twin pair were both more likely to have chosen each other as their closest friend when compared to $\mathrm{DZ}$ ss or DZ ms twins. While DZ ss twins were just as likely to fall into any of the three reciprocity categories, DZ $\mathrm{ms}$ twin pairs were more likely to choose someone other than their co-twin as their closest friend (see Table 2).

Two independent $t$ tests were performed to examine whether twins who chose each other as their closest friend reported significantly higher levels of total intimacy with their co-twin when compared to twins where only one member chose the other as closest friend, or when neither twin chose the other as closest friend. The first $t$ test demonstrated that twins who both chose each other as their closest friend reported significantly higher levels of intimacy with their co-twin $(M=5.66)$, when compared to twins where only one member of the pair chose the other as their closest friend $[M=5.26 ; t(155)=-3.34, p<.01]$. The second $t$ test indicated that twins who both chose someone other than their co-twin as their closest friend reported significantly lower levels of intimacy with their co-twin $(M=$ 4.44) than twins where only one twin chose his/her co-twin as closest friend $[M=5.26 ; t(97)=-5.52, p<.001]$. Thus, the highest level of intimacy was reported by twins who both nominated each other as closest friend, followed by twins where only one twin nominated the other as closest friend, and finally twins where neither twin nominated the other as closest friend.

Table 2

Percentage of MZ, DZ ss, and DZ ms Twins in Each of the Three Reciprocity Categories

\begin{tabular}{|c|c|c|c|}
\hline & \multicolumn{3}{|c|}{ Zygosity' } \\
\hline & $\mathrm{MZ}$ & DZ ss & $\mathrm{DZ} \mathrm{ms}$ \\
\hline \multicolumn{4}{|l|}{ Reciprocity with Co-twin } \\
\hline Both twins chose the other as closest friend & $70.3 \%$ & $31.0 \%$ & $8.3 \%$ \\
\hline One twin chose the other as closest friend & $17.2 \%$ & $38.0 \%$ & $25.0 \%$ \\
\hline Neither twin chose the other as closest friend & $12.5 \%$ & $31.0 \%$ & $66.7 \%$ \\
\hline
\end{tabular}

Note. ${ }^{1}$ The sample size varied according to zygosity for each cell. Overall, $128 \mathrm{MZ}$ twins, $58 \mathrm{DZ}$ twins, and $24 \mathrm{DZ}$ ms twins were included in this analysis. 


\section{Level of Intimacy with Co-Twin}

Four independent $t$ tests were performed to evaluate whether twins who reported high levels of intimacy with their co-twin reported lower levels of intimacy with their closest SS and closest OS friend when compared to twins who reported low levels of intimacy with their co-twin. For this analysis, twins were classified according to their total intimacy scores with their co-twin. Overall, 18.7\% (n $=40$ ) reported High levels of intimacy (scores between 6-7), 66.8\% ( $n=143)$ reported Average levels of intimacy (scores between $4-5)$, and $9.3 \%(n=20)$ reported Low levels of intimacy (scores between 1-3) with their co-twin; $5.1 \%(n=11)$ were missing too much data to be included. No significant differences were found between any of the groups. Thus, the reported level of intimacy with co-twin was not related to significantly higher or significantly lower levels of reported intimacy with closest SS friend or closest OS friend.

\section{Difference Scores Across Relationships}

Three paired $t$ tests were performed to examine whether a greater discrepancy in total intimacy scores occurred between co-twin and closest OS friend when compared to co-twin and closest SS friend. Difference scores were calculated by subtracting total intimacy scores for closest OS and closest SS friend (respectively) from total intimacy scores with co-twin. This was done for $\mathrm{MZ}, \mathrm{DZ}$ ss, and $\mathrm{DZ}$ ms twins. All three paired $t$ tests found significant differences. A greater discrepancy in total intimacy scores occurred between co-twin and closest OS friend when compared to co-twin and closest SS friend for $\mathrm{MZ}[t(90)=4.20, p<$ $.001]$, DZ ss $[t(44)=2.74, p<.01]$, and DZ ms twins $[t(18)=3.97, p<.01]$. See Table 3 .

It is important to note that while positive difference scores were found for $\mathrm{MZ}$ and $\mathrm{DZ}$ ss twins (indicating that intimacy with co-twin was higher), a negative difference score was found between co-twin and closest OS friend for $\mathrm{DZ}$ ms twins, indicating that DZ ms twins reported higher levels of intimacy with their closest SS friend compared to their co-twin. Finally, a significant positive correlation was found between each of the three pairs of difference scores, indicating that as the intimacy levels between co-twin and closest SS friend became more similar, the intimacy levels between co-twin and closest SS friend also became more similar for MZ $(r=.80, p<.001)$, DZ ss $(r=.63, p<.001)$ and DZ ms twins $(r=.61, p<.01)$.

\section{Difference Scores Across Zygosity}

Four independent $t$ tests were performed to examine whether the difference in total intimacy scores between cotwin and closest OS friend, and co-twin and closest SS friend were greater for $M Z$ twins when compared to $\mathrm{DZ}$ ss and DZ ms twins, respectively. The difference in total intimacy scores between co-twin and closest SS friend were significant only when comparing DZ ss with DZ ms twins $[t(79)=3.39, p<.001]$, where DZ ss twins showed significantly greater differences between co-twin and closest SS friend (this remains significant after using the Bonferroni procedure to control for the familywise type 1 error rate). Although MZ twins showed greater differences in intimacy levels in all three relationships when compared to DZ ss and DZ ms twins respectively, these differences were not significant (see Table 4).

\section{Exploratory Analyses}

Age Trends and Total Intimacy Scores

A repeated measures MANOVA was performed with Age as the between-subjects variable and Relationship Type (total intimacy scores for closest SS friend, closest OS friend and co-twin) as the within-subjects variable. A non significant main effect of Age was found, indicating that twins did not report different levels of total intimacy for closest SS friend, closest OS friend or co-twin as a function of Age. Significant within-subject effects were found, however, for Relationship Type $[F(1,151)=33.07, p<$ $\left..001, \eta^{2}=.18\right]$ and Relationship Type X Age $[F(3,151)=$ 4.37, $\left.p<.01, \eta^{2}=.08\right]$.

Four repeated measures MANOVAs were performed on total intimacy scores using closest SS friend, closest OS friend, and co-twin as the within-subjects variable, to examine how intimacy varies across the three Relationship Types, while holding Age constant. Significant withinsubject differences across the three relationship types were found for the two middle age categories: young adulthood

\section{Table 3}

Comparing Mean Difference Scores ${ }^{1}$ Between Co-twin and Closest OS Friend, and Co-twin and Closest SS Friend for MZ, DZ ss and DZ ms Twins

\begin{tabular}{|c|c|c|c|}
\hline & \multicolumn{3}{|c|}{ Difference Scores } \\
\hline & $M_{D}$ & $S D_{\mathrm{D}}$ & $N$ \\
\hline \multicolumn{4}{|c|}{ Comparing Twins on Total Intimacy Scores (TIS) Between Relationships } \\
\hline \multicolumn{4}{|c|}{ MZ Twins } \\
\hline TIS twin - TIS OS & .90 & 1.31 & 91 \\
\hline TIS twin - TIS SS & .55 & 1.04 & 91 \\
\hline \multicolumn{4}{|l|}{ DZ ss Twin } \\
\hline TIS twin - TIS OS & .70 & .92 & 45 \\
\hline TIS twin - TIS SS & .37 & .96 & 45 \\
\hline \multicolumn{4}{|l|}{ DZ ms Twins } \\
\hline TIS twin - TIS OS & .25 & .80 & 19 \\
\hline TIS twin - TIS SS & -.34 & .60 & 19 \\
\hline
\end{tabular}


Table 4

Comparing MZ, DZ ss, DZ ms Twins on Mean Difference Scores' Between Co-twin and Closest OS Friend, and Between Co-twin and Closest SS Friend

\begin{tabular}{lcc}
\hline & & Difference Scores \\
& $M_{\mathrm{D}}$ & $S D_{\mathrm{D}}$ \\
\hline Comparing Difference Scores Across Twin Type & & \\
Co-Twin - Closest OS Friend & & \\
MZ & .95 & .32 \\
DZ ss & .70 & .92 \\
DZ ms & .25 & .80 \\
Co-Twin - Closest SS Friend & 45 & 19 \\
MZ & .63 & .99 \\
DZ ss & .42 & .99 \\
DZ ms & -.32 & .59 \\
\hline
\end{tabular}

Note. 'Mean difference scores were calculated by subtracting total intimacy scores for closest other- sex (OS) friend and closest same-sex (SS) friend (respectively) from total intimacy scores with co-twin. This was done for MZ, DZ ss, and DZ ms twins.

[20-35 years of age; $\left.F(1,68)=34.57, p<.001, \eta^{2}=.34\right]$ and middle adulthood [36-50 years of age; $F(2,60)=$ 32.38, $\left.p<.001, \eta^{2}=.50\right]$. Since Mauchley's Test of Sphericity was significant for the young adulthood age category $\left[\chi^{2}(2)=16.94, p<.001\right]$, the Lower-bound epsilon (.50) was used to adjust the degrees of freedom in both the numerator and the denominator. Mauchley's Test of Sphericity was not significant for the middle adulthood age category, therefore the Greenhouse-Geisser epsilon (.91) was used to adjust the degrees of freedom in both the numerator and the denominator.

Significant within-subject differences across the three Relationship Types were not found for the youngest (adolescence) or oldest (older adulthood) age groups. Post hoc analyses using Scheffé's $F$-statistic found that twins in young and middle adulthood reported significantly higher levels of intimacy with co-twin when compared to closest SS friend $[F(1,68)=9.91, p<.01 ; F(2,60)=13.19, p<$ .001 ; in young and middle adulthood respectively]. In addition, twins in these two age groups also reported signif-

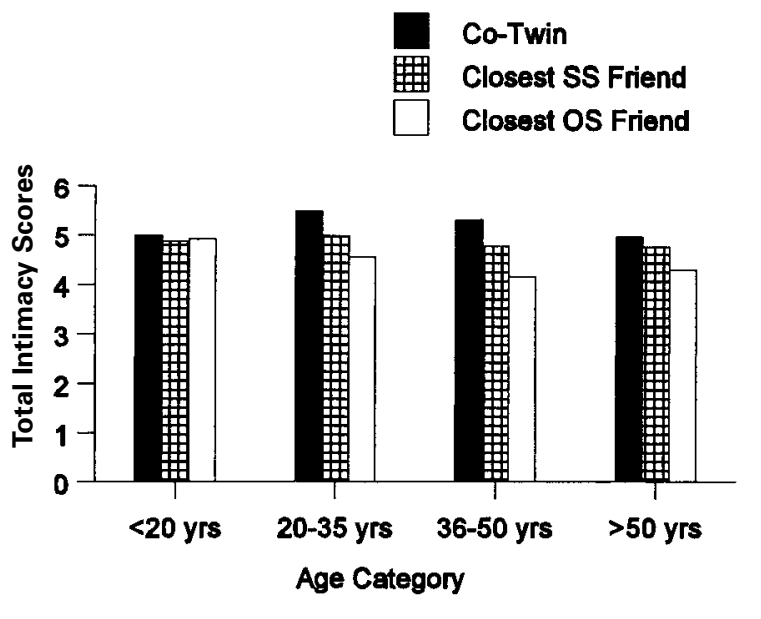

\section{Figure 1}

Differences in reported intimacy levels within the three relationship types as a function of age. icantly higher levels of intimacy with closest SS friend compared to closest OS friend $[F(1,68)=7.68, p<.01 ; F(2$, $60)=16.76, p<.001$; in young and middle adulthood respectively]. See Figure 1.

Overall, total intimacy scores with co-twin were higher than total intimacy scores with closest SS friend and closest OS friend in every age category (although not significantly higher in adolescence or older adulthood). Furthermore, while total intimacy scores with closest SS friend were higher than closest OS friend in the three oldest categories (young adulthood, middle adulthood and older adulthood), adolescent twins reported higher (though non significant) levels of intimacy with their closest OS friend compared to their closest SS friend. It is also interesting to note that adolescents and older adults reported levels of intimacy that were similar across the three relationship types, while twins in young and middle adulthood reported significantly different levels of intimacy in each of these three relationships.

\section{Examining the Dimensions of Intimacy}

A 3 (Zygosity) by 2 (Sex) MANOVA was performed on the eight dimensions of intimacy for closest SS friend, closest OS friend and co-twin to determine how these components varied as a function of Zygosity and/or Sex in each of the three relationships. Significant effects for each of the eight dimensions were not found as a function of Zygosity, Sex, or Zygosity X Sex for any of the three relationship types. Thus, MZ twins did not report significantly higher levels of Exclusiveness, Trust/Loyalty, Common Activities, Frankness/Spontaneity, Sensitivity/Knowing, Giving/ Sharing, Imposing/Taking, or Attachment in any of the three relationships when compared to DZ ss or DZ ms twins. Furthermore, females did not report significantly higher levels of intimacy on any the eight dimensions with co-twin, closest SS friend, or closest OS friend when compared to males.

\section{Predicting Intimacy with Closest Friends}

Two standard multiple regressions were performed to examine whether scores on any of the eight dimensions of intimacy with co-twin predicted total intimacy scores with 
closest SS and/or closest OS friend. In the first regression, total intimacy scores with closest SS friend were entered as the criterion variable and the average scores on each of the eight dimensions for co-twin were the predictors. The second regression included total intimacy scores with closest OS friend as the criterion variable and the average scores on each of the eight dimensions of intimacy with cotwin were the predictors. It was predicted that Exclusiveness ratings in twinship would be the most powerful predictor of low intimacy between twins and their closest same- and other-sex friend.

In the first analysis, significant zero-order correlations were found between total intimacy scores with closest SS friend and four of the eight co-twin predictors (Exclusiveness, $r=.17, p<.01$; Common Activities, $r=$ $.15, p<.05$; Sensitivity/Knowing, $r=.19, p<.01$; and Giving/Sharing, $r=.20, p<.01)$. Although $R_{\text {adj }}(.24)$ for the regression was significant $[F(8,193)=2.53, p<.05]$, Giving/Sharing and Sensitivity/Knowing were the only two significant predictors. Since the standard method of multiple regression used in this analysis was highly sensitive to the unique contribution of each predictor, the significant correlations found among each of the predictors indicates that many of the predictors shared a large degree of their variance (average correlation among predictors = .47 , range $=.14$ to .66 ). As a result, the predictive strength of the predictors might have been mediated by, or made redundant as a result of their strong correlations with the other predictor variables.

In the second multiple regression, a significant zeroorder correlation was found between total intimacy scores with closest OS friend and one of the eight co-twin predictors (Exclusiveness, $r=.16, p<.05)$. A significant regression equation was not found $[F(8,149)=1.64, p=$ $.12]$, however, as was the case in the first regression, significant correlations were found among all of the predictors (average correlation among predictors $=.51$, range $=.20$ to .69). It is important to note that in both regressions, the significant correlations between the criterion and the predictor variables were all positive. Thus, higher levels of intimacy with co-twin on these dimensions were related to higher levels of total intimacy with closest SS and closest OS friend.

\section{$\overline{\text { Discussion }}$ \\ Intimacy with Co-twin}

Although the twins in the current study reported high levels of intimacy with their co-twin, these levels did not differ as a function of zygosity. Thus, the expectation that $\mathrm{MZ}$ twins would report significantly higher levels of intimacy with their co-twin when compared to DZ ss or DZ ms twins was not supported. Not only was this found when using total intimacy scores, but it was also encountered for each of the eight intimacy dimensions. These results were quite different from what was expected and from what has been found previously. As indicated in the introduction, research has found that MZ twins spend significantly more time together, indicate that they and their co-twin are inseparable, and may be more likely to value their twinship due to their similar appearance and their similar treatment by others. Nevertheless, higher levels of intimacy on dimensions related to shared activities (Common Activities, Exclusiveness) and attachment to one another (Attachment) were not found. It is important to keep in mind, however, that much of the twin literature has focused on twins and their social interactions during infancy, childhood and early adolescence, while this study examined twins from late adolescence to late adulthood. Therefore, it is possible that the $\mathrm{MZ}$ twins in this sample would have reported higher levels of intimacy with their co-twin if they had been surveyed earlier. Perhaps the difference between twins and their relationships with each other changes as a function of developmental stage.

Nevertheless, members of a MZ twin pair were both more likely to nominate each other as their closest friend when compared to DZ ss and DZ ms twins. As expected, members of a twin pair who nominated each other as their closest friend reported significantly higher levels of intimacy with their co-twin when compared to twins where only one twin nominated the other as closest friend, or where neither twin nominated the other as closest friend. While DZ ss twins were just as likely to fall into any of the three reciprocity categories (Both, One, and Neither), DZ ms twins were more likely to choose someone other than their co-twin as their closest friend. Thus, while zygosity did not directly influence reported levels of intimacy with co-twin, twins who chose each other as their closest friend reported higher levels of intimacy with their co-twin, and these twins were more likely to be MZs.

\section{Intimacy with Co-twin, Closest SS Friend, and Closest OS Friend}

Contrary to expectation, twins who reported higher levels of intimacy with their co-twin did not report significantly lower levels of intimacy with their closest SS or closest OS friend. This trend was reported for twins in general as well as for MZ, DZ ss and DZ ms twins. Thus, while researchers have concluded that some of the isolating behaviors observed in twins during childhood are linked to the twin relationship (e.g., twins are less likely to engage in social activities with others, prefer solitary play, or exhibit different/less mature styles of play; Cohen et al., 1977; Savic, 1980; Vandell et al., 1988), this study does not support the conclusion that having an intimate twin relationship interferes with the formation of close relationships with others during late adolescence or throughout adulthood. More specifically, while MZ twins were perceived to be at higher risk for maintaining a socially exclusive twin relationship, they did not report significantly lower levels of general intimacy, or lower levels of intimacy on any of the eight intimacy dimensions with their closest SS or closest OS friend. Furthermore, differences were not found between same-sex twins (MZ, DZ ss) when compared to mixed-sex twins (DZ ms). These findings are in line with the belief that the existence of one satisfactory and highly intimate relationship does not necessarily occur at the expense of others (Collins \& Repinski, 1994). Nevertheless, this study examined twin relationships from a cross sectional perspective. A longitudinal design would be needed 
in order to address this question from a developmental viewpoint.

In agreement with the literature on singletons, the twins in the current study reported significantly higher levels of general intimacy with their closest SS friend when compared to their closest OS friend. However, when these relationships were examined as a function of zygosity, a slightly different trend appeared. Although MZ and DZ ss twins reported levels of intimacy that were more similar between co-twin and closest SS friend (than co-twin and closest OS friend), DZ ms twins reported intimacy levels that were more similar between co-twin and closest OS friend (than co-twin and closest SS friend). Although confusing at first, this finding indicates that DZ ms twins reported that their relationship with their other-sex co-twin was more similar to their relationship with their closest OS friend than with their closest SS friend. Furthermore, while MZ and DZ ss twins reported higher levels of intimacy with their co-twin than with either their closest SS or closest OS friend, DZ ms twins reported the highest level of intimacy with their closest SS friend. Thus, in cases where one's co-twin is of the other sex, the qualities important in a same-sex relationship may not be fulfilled in the twinship, and are therefore found in another relationship (i.e., with closest SS friend).

It was also predicted that $M Z$ twins would report the greatest difference in total intimacy scores between their cotwin and closest OS friend, and that DZ ms twins would report the smallest difference in total intimacy scores between their co-twin and closest OS friend. Although this trend was observed, a significant difference was only found between DZ ss and DZ ms twins where DZ ss twins reported significantly larger differences in intimacy between their co-twin and closest SS friend when compared to DZ $\mathrm{ms}$ twins. However, since the difference score for DZ ms twins was negative for intimacy levels between co-twin and closest SS friend, this finding might have been a consequence of the way the difference score was calculated, rather than the absolute difference between the scores.

\section{Predicting Intimacy in Friendship From Twinship}

While it was anticipated that higher levels of Exclusiveness within the twin relationship would predict lower levels of intimacy with closest SS friend and closest OS friend, this was not supported in the current study — in fact, the opposite trend was found. Twins who reported high levels of Exclusiveness with their co-twin (i.e., prefer to be alone together), reported more intimate relationships with close others. Perhaps then, substantial amounts of one-on-one time with one another over extended periods of time allows twins to gain a better understanding of how to relate to close others. Moreover, the results also indicate that as levels of Sensitivity/Knowing, Giving/Sharing, and Common Activities increased in the twin relationship, levels of total intimacy with closest SS friend also increased. Therefore relationship skills surrounding high levels of empathy, understanding, helping, as well as shared activities and experiences in the context of the twin relationship, benefit twins' relationships with their closest SS friend.

\section{Male/Female Differences in Intimacy}

In line with the literature on singletons, it was predicted that females would report higher levels of intimacy across all three relationship types when compared to males. Although these trends were found, a significant difference between males and females was only observed for closest SS friend, where females reported significantly higher levels of intimacy when compared to males. Furthermore, when females and males were compared on the eight intimacy dimensions, significant differences were not found for any of the three relationships. Thus, these results tend to indicate that there may be something unique about the twin relationship that transcends general intimacy trends found in male and female singletons. Perhaps the difference is a result of family ties and prolonged periods of interaction with the same individual. Furthermore, it may be that relationships involving women tend to be more intimate (women's relationships with women and men's relationships with women). It would be interesting to compare reported levels of intimacy in males with their co-twin, non-twin sibling and male friend, with male singleton's relationships, to see how these differ.

\section{Age and Intimacy}

Although twins did not report different levels of total intimacy for closest SS friend, closest OS friend or co-twin as a function of age, significant differences were found across the three different relationships during early and middle adulthood (20-35, 36-50) where significantly higher levels of intimacy were reported with co-twin, followed by closest SS friend and closest OS friend respectively. While the highest level of intimacy was reported with co-twin across the three relationships, it was significantly higher in young and middle adulthood only. Since individuals are more likely to turn to their partners and their family members for help and support than to their friends during times of distress (Eshel et al., 1998; Jamieson, 1998), perhaps twins approach one another when it seems inappropriate to ask friends for practical or instrumental assistance. On the other hand, it may be that individuals in adolescence and late adulthood have more time to invest in a wider variety of relationships and to develop equally intimate relationships with their twin and close friends. These two developmental periods may reflect a time when adolescents are immersed in their peer network, and when the changing family roles of older adults provide more opportunities to reconnect with friends.

\section{Limitations}

While this study has contributed to the current literature on twins and their relationships with others, certain limitations should be kept in mind when interpreting its findings. Although an attempt was made to recruit equal numbers of males and DZ twins, more females and MZs participated in the current study. Therefore, analyses examining intimacy as a function of zygosity might have been more powerful if the sample had included equal numbers of males and females as well as $\mathrm{MZ}, \mathrm{DZ}$ ss and DZ ms twins. Moreover, since the majority of the sample were young single adults with a college or university education, 
the representativeness of the sample and the generalizability of the results are somewhat questionable.

Although the current study found that twins did not differ from each other in terms of the intimate relationships they shared with close others, it is still unclear how these twins compare with non-twins. Not only would the inclusion of a control group add confidence to future findings, but it would also expand the knowledge of how twin relationships are different and how they are the same as singletons' relationships — including sibling relationships, parent-child relationships, peer relationships, and romantic relationships. Furthermore, while the current study examined whether or not levels of intimacy within the twinship influence twins' relationships with others, it is unclear how relationships with others influence the twin relationship. Consequently, it would be interesting to see how dating relationships, marital partners, and the birth of a child influence the twin bond.

Previous research has found that intimate relationships have a greater impact on relationships with more social distance (acquaintances, casual friends) when compared to relationships with less social distance (close friends, partners, family members; Milardo et al., 1983). Since the current study examined twins' relationships with close others, the impact of the twin relationship might have been lost on more peripheral relationships. Therefore, in order to increase our confidence in these findings, an examination of the broader peer network should be conducted.

\section{Implications and Conclusions}

By using a direct approach in the examination of twins' relationships with their co-twins and close others, it has been found that the twin relationship may actually benefit twins rather than harm them. While previous research has focused on the disadvantages of being a twin and the hazards that the "twinning reaction" may bear, few studies have ventured to examine the positive implications of such a close and enduring relationship. Indeed, twins may have a lot to teach us about seemingly "exclusive" relationships and the development of future social functioning.

Although higher levels of intimacy are generally reported between twins, the twin relationship does not appear to differ significantly from other close relationships on any of the eight dimensions of intimacy measured here. Nevertheless, the absence of significant sex differences between male and female twins in their relationships with others warrants future research. In fact, the twin situation has the potential to provide an interesting and informative method of studying a combination of close relationships between siblings, age mates, and different sexes across time. Finally, while twins encounter their fair share of the trials and tribulations involved with close relationships, the end result appears to be positive.

\section{References}

Adelman, M. B., \& Siemon, M. (1986). Communicating the relational shift: Separation among adult twins. American Journal of Psychotherapy, XL(1), 96-109.

Brothers, B. J. (Ed.). (1991). Intimate autonomy: Autonomous intimacy. New York: The Haworth Press.
Buhrmester, D., \& Furman, W. (1986). The changing functions of friends in childhood: A neo Sullivan perspective. In V. J. Derlega, \& B. A. Winstead (Eds.), Friendship and social interaction (pp. 41-62). New York: Springer-Verlag.

Clark, P.M., \& Dickman, Z. (1984). Features of interaction in infant twins. Acta Geneticae Medicae et Gemellologiae: Twin Research, 33, 165-171.

Cohen, D. J., Dibble, E., \& Grawe, J. M. (1977). Fathers' and mothers' perceptions and children's personality. Archives of General Psychiatry, 34, 480-487.

Collins, W. A., \& Repinski, D. J. (1994). Relationships during adolescence: Continuity and change in interpersonal perspective. In R. Montemayor, G. R. Adams, \& T. P. Gullotta (Eds.), Personal relationships during adolescence. Advances in adolescent development, annual book series no. 6. London: Sage.

Eshel, Y., Sharabany, R., \& Friedman, U. (1998). Friends, lovers and spouses: Intimacy in young adults. British Journal of Social Psychology, 37, 41-57.

Jamieson, L. (1998). Intimacy: Personal relationships in modern society. Cambridge: Polity Press.

Jones, G. P., \& Dembo, M. H. (1989). Age and sex role differences in intimate friendships during childhood and adolescence. Merrill-Palmer Quarterly, 35(4), 445-462.

Kasriel, J., \& Eaves, L. (1976). The zygosity of twins: Further evidence on the agreement between diagnosis by blood groups and written questionnaires. Journal of Biosocial Science, 8, 263-266.

Mayseless, O. (1993). Gifted adolescents and intimacy in close same-sex relationships. Journal of Youth and Adolescence, 22(2), $135-146$.

Mayseless, O., Sharabany, R., \& Sagi, A. (1997). Attachment concerns of mothers as manifested in parental, spousal, and friendship relationships. Personal Relationships, 4, 255-269.

Mayseless, O., Wiseman, H., \& Hai, I. (1998). Adolescents' relationships with father, mother, and same-gender friend. Journal of Adolescent Research, 13(1), 101-123.

Milardo, R. M., Johnson, M.P., \& Houston, T. L. (1983). Developing close relationships: Changing patterns of interaction between pair members and social networks. Journal of Personality and Social Psychology, 44, 964-976.

Nichols, R. C., \& Bilbro, W. C. Jr. (1966). The diagnosis of twin zygosity. Acta Geneticae Medicae et Gemellologiae, 16, $265-275$.

Pearlman, E. M. (1990). Separation-individuation, self-concept, and object relations in fraternal twins, identical twins, and singletons. The Journal of Psychology, 124(6), 619-628.

Sandbank, A. C. (1988). The effect of twins on family relationships. Acta-Geneticae Medicae et Gemellologiae, 37(2), $161-171$

Savic, S. (1980). How twins learn to talk: A study of the speech development of twins from 1 to 3. New York: Academic Press.

Segal, N. L. (1999). Entwined lives: Twins and what they tell us about human behavior. New York, NY: Dutton Book.

Sharabany, R. (1974). Intimate friendships among kibbutz and city children and its measurement. Ph.D. Dissertation, Cornell University. Dissertation Abstracts International, 35(2), 10289B. 
Sharabany, R. (1994a). Intimate friendship scale: Conceptual underpinnings, psychometric properties and construct validity. Journal of Social and Personal Relationships, 11, 449-469.

Sharabany, R. (1994b). Continuities in the development of intimate friendships: Object relations, interpersonal and attachment perspectives. In R. Erber, \& R. Gilmour (Eds.), Theoretical frameworks for personal relationships (pp. 157-178). Hillsdale, NJ: Lawrence Erlbaum Associates Publishers.

Sharabany, R. Gershoni, R., \& Hofman, J. E. (1981). Girlfriend, boyfriend: Age and sex differences in intimate friendships. Developmental Psychology, 17(6), 800-808.

Siemon, M. (1980). The separation-individuation process in adult twins. American Journal of Psychotherapy, 34, 387-400.
Tambs, K., Sundet, J. M., \& Berg, K. (1985). Cotwin closeness in monozygotic and dizygotic twins: A biasing factor in IQ heritability analysis? Acta Geneticae Medicae et Gemellologiae: Twin Research, 34(1-2), 33-39.

Vandell, D. L., Owen, M. T., Wilson, K. S., \& Henderson, V. K. (1988). Social development in infant twins: Peer and motherchild relationships. Child Development, 59, 168-177.

Vandenberg, S. G. (1984). Does a special twin situation contribute to similarity for abilities in $\mathrm{MZ}$ and $\mathrm{DZ}$ twins? Acta Geneticae Medicae et Gemellologiae: Twin Research, 33(2), 219-222.

Zazzo, R. (1976). The twin condition and the couple effects on personality development. Acta Geneticae Medicae et Gemellologiae: Twin Research, 25, 343-352.

\section{CALL FOR PAPERS}

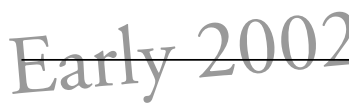

Special Issue on Bereavement in Twins

GUEST EDITOR: Elizabeth Bryan, London

This special issue will be published early in $\mathbf{2 0 0 2}$ so time is running out to contribute.

Please email Elizabeth Bryan at ebryan@higgins7.co.uk as soon as possible if you wish to contribute.

\section{Time is Running Out}

\section{CALL FOR PAPERS}

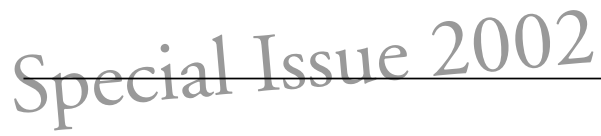

Special Issue on Twin Registers

GUEST EDITOR: Andreas Busjahn, Berlin

Following the successful workshop on twin registers at this year's ISTS conference in London,

Twin Research will publish a special issue devoted to an overview of active registers throughout the world.

If you would like an overview of your twin register to be included then please contact the guest editor for this issue, Dr Andreas Busjahn at a_busjahn@yahoo.com as soon as possible. An added incentive is that the material gathered will be collated for a review in Nature Reviews: Genetics in 2002 to illustrate the tremendous resource for human gene mapping available from twin registers.

Andreas has also implemented an email group at twinregisters-subscribe@yahoogroups.com for the exchange of ideas and experiences concerning running twin registers. 